\title{
Pneumothorax and Pneumatocoele Formation in a Patient with COVID-19: a Case Report
}

\author{
P. Capleton ${ }^{1} \cdot$ W. Ricketts ${ }^{2} \cdot$ K. Lau $^{3} \cdot$ S. Ellis ${ }^{4} \cdot$ M. Sheaff ${ }^{1} \cdot$ K. Giaslakiotis ${ }^{1} \cdot$ S. Uys ${ }^{2} \cdot$ Nairi Tchrakian $^{1}$ (D)
}

Accepted: 2 December 2020 / Published online: 7 January 2021

(C) The Author(s), under exclusive licence to Springer Nature Switzerland AG part of Springer Nature 2021

\begin{abstract}
Coronavirus disease 2019 (COVID-19) causes significant morbidity and mortality for a proportion of infected patients, and our knowledge and understanding of its clinical, radiological and histopathological features are still evolving. An association between COVID-19 and pneumothorax has been described in an increasing number of case reports and series in the literature, which have largely focused on clinical and imaging features. We report the case of a patient who developed COVID-19 complicated by pneumothorax, requiring surgical intervention. We describe the histopathological features seen in the thorascopically resected bullectomy specimen - this is, to our knowledge, the first reported description of the morphological features of pneumothorax in this important clinical setting.
\end{abstract}

Keywords COVID-19 $\cdot$ Morphology $\cdot$ Pneumothorax $\cdot$ Pneumatocoele $\cdot$ SARS-CoV-2

\section{Introduction}

Coronavirus disease 2019 (COVID-19) is an infectious disease caused by severe acute respiratory syndrome virus 2 (SARS-CoV-2). It causes significant morbidity and mortality for a proportion of those infected and has been declared a global pandemic by the World Health Organization (WHO). The occurrence of pneumothorax in the setting of COVID-19 has been described in several case reports and occasional series in the literature to date [1-5], but the association between the two has yet to be elucidated. Typical clinical and radiological features of COVID-19 have been detailed since early in the pandemic [6,7]; however, the histopathological features of the infection are less well described. Similarly, the

This article is part of the Topical Collection on Covid-19

Nairi Tchrakian

nairitchrakian@gmail.com

1 Department of Pathology, The Royal London Hospital,, Barts Health NHS Trust, London, UK

2 Department of Respiratory Medicine, Barts Thorax Centre, Barts Health NHS Trust, London, UK

3 Department of Thoracic Surgery, Barts Thorax Centre, Barts Health NHS Trust, London, UK

4 Department of Diagnostic Imaging, Barts Health NHS Trust, London, UK descriptions of pneumothorax in this setting have largely centred on clinical and imaging findings, but specific histopathological features have, to our knowledge, not yet been reported in a focused fashion.

Here, we report the case of a patient who, following a prolonged hospital admission, developed COVID-19 complicated by a pneumothorax necessitating surgical management due to a persistent air leak. We describe the histopathological features in the surgical resection specimen and also briefly discuss the potential mechanisms that could give rise to development of pneumothorax in the setting of COVID-19 infection.

\section{Case Report}

\section{Clinical Presentation}

The patient, a 64-year-old female non-smoker with a number of comorbidities, including previous cANCA vasculitis necessitating renal transplantation, hypertension and right middle lobe bronchiectasis, had been admitted for over 2 months for respiratory failure due to Staphylococcus aureus bacteraemia and pneumonia, during which time she required intubation and ventilation for presumed Pneumocystis jirovecii infection and cytomegalovirus viraemia. She was undergoing rehabilitation when she developed a low-grade fever and desaturation. A chest X-ray showed a $3-\mathrm{cm}$ right-sided pneumothorax, and 
a nasopharyngeal swab for COVID-19 RNA returned a positive result. CT showed tethering of the lung associated with a cystic lesion/bulla suggestive of a pneumatocoele but no typical features of COVID-19 (Fig. 1a). Despite insertion of a chest drain, the pneumothorax persisted.

She recovered from COVID with negative nasopharyngeal swabs and underwent successful video-assisted thoracoscopic surgery (VATS) and wedge resection of the tethered cystic area which was found to be bubbling at operation. Despite the multiple negative nasopharyngeal swabs, an intraoperative bronchoalveolar lavage (BAL) tested positive for COVID-19 RNA.

\section{Pathology Examination}

Macroscopic examination of the resected lung wedge showed focal pleural fibrosis, and sectioning revealed a $15-\mathrm{mm}$ cystic space within the parenchyma with a smooth inner surface. There were no other gross abnormalities. Microscopically, the wall of the cystic space was densely fibrotic and lined by simple cuboidal epithelium (Figs. 1b and 2a). The cyst wall was seen to transition into the respiratory epithelium of a dilated airway, suggesting connection of the cyst with the bronchial tree (Fig. 2b), in keeping with the radiological impression of a pneumatocoele. There was no obvious connection seen histologically between the cystic lesion and the pleura to explain the persistent air leak. However, it may have been that the air leak site was not represented in the sections submitted for histology, either due to sampling or being situated in or adjacent to the specimen staple line.

Sections from the background lung showed areas of collapse interspersed with aerated lung, as well as subpleural and septal fibrosis with oedema and vascular congestion (Fig. 3a). There was mild patchy parenchymal and peribronchial inflammation associated with pneumocyte hyperplasia. Neutrophils and eosinophils were not a conspicuous component of the inflammatory infiltrate. Viral cytopathic change was not seen, and no granulomas were identified. Occasional dilated airways were noted, in keeping with the stated history of bronchiectasis. Some airspaces contained collections of fibrin with accompanying haemosiderin, and scattered intra-alveolar fibromyxoid plugs were identified, possibly representing early reparative change (Fig. 3b). There was no evidence of hyaline membrane disease. Peripheral emphysematous change was present, but no bullae were identified.

\section{Discussion}

Many of the descriptions of the histopathological features of COVID-19 infection found in the literature at the time of writing relate to autopsy (or post-mortem needle biopsy) findings in patients who died of severe disease - chiefly diffuse alveolar damage (DAD) in an acute respiratory distress syndrome (ARDS) setting [8-11], which itself has a number of infectious and non-infectious aetiologies. However, a recent paper has reported similar findings to ours in two lobectomy specimens from patients subsequently found to be COVID19-positive, and therefore are judged likely to represent early changes of the disease, namely, vascular congestion, mild inflammation without a significant acute component and pneumocyte hyperplasia [12]. Prominent intra-alveolar oedema and proteinaceous exudates were also described, which were not features seen in our case. Possible viral cytopathic changes within pneumocytes have been reported in several cases $[8,10,12]$ but have not been well-characterized in the literature to date.

An association between COVID-19 and pneumothorax has been described, but the mechanisms underlying this association have yet to be elaborated. Martinelli et al. who co-ordinated a recent multicentre study (the largest series described in the literature to date) analysing this clinical scenario conclude that the relative frequency of this copresentation of pathologies indicates that it is unlikely to be coincidental [13]. While it has been previously suggested that the development of pneumothorax may
Fig. 1 a Axial CT image demonstrating a right-sided pneumothorax with stranding evident in the pleural space; a cystic lesion in the periphery of the lung parenchyma is also present, which shows demonstrable communication with the pleural space, compatible with pneumatocoele. b Low power view of the cystic space demonstrating the densely fibrotic wall with surrounding vascular congestion (reprinted with permission from the European Respiratory Journal)
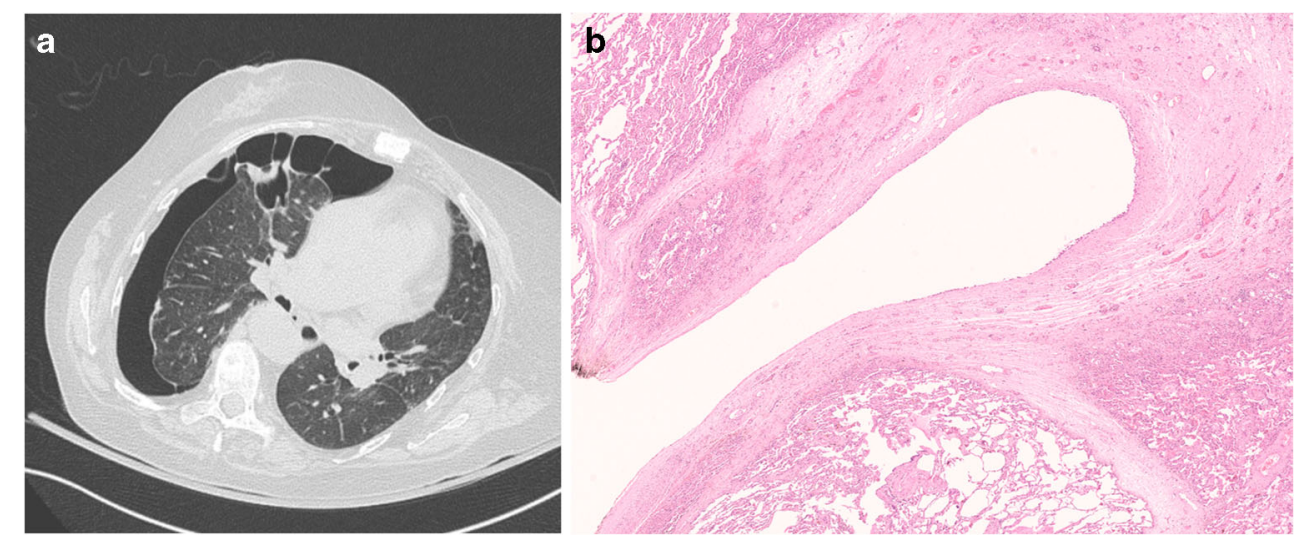


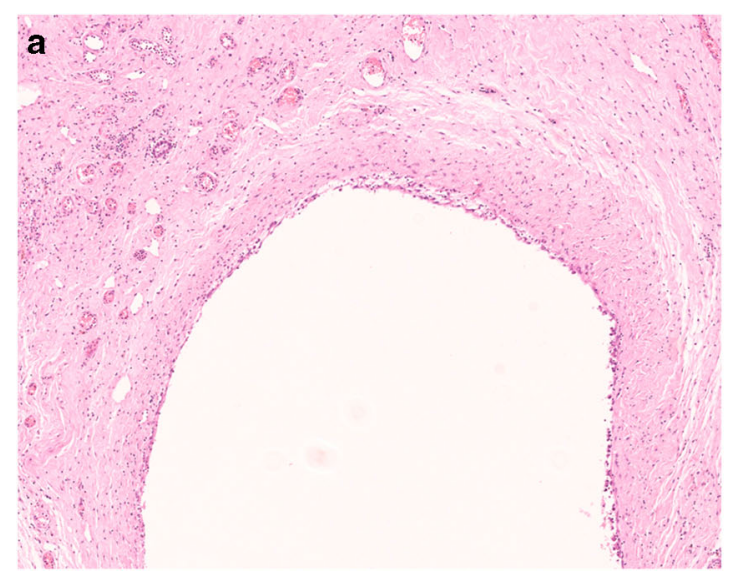

Fig. 2 a Intermediate power view of the cystic space exhibiting a simple cuboidal epithelial lining and fibrotic wall associated with vascular congestion. b Intermediate power image of the fibrous cyst wall (right)

correlate with disease progression [6], the above study by Martinelli et al. suggests that it does not seem to be a marker of poor prognosis in this setting, and the authors encourage active treatment where clinically possible.

Aiolfi et al., in a paper outlining their approach to the surgical management of COVID-19 patients with pneumothorax, attribute the occurrence of pneumothorax in these patients to a combination decreased lung compliance (due to the histological changes described above) and sustained pressure ventilation, which they postulate results in rupture of pre-existing blebs [2]. It is felt, however, that barotrauma alone cannot account for the co-occurrence of pneumothorax in COVID19 infection [13]. Liu et al. described two reports of cystic lung disease in COVID-19 patients - one of whom suffered pneumothorax - the authors suggest that the fibromyxoid exudates which develop in this infection can predispose to pneumothorax through pressure differentials resulting from a ball/ valve mechanism within airways and airspaces [14]. It is also possible that the mechanical effect of a persistent dry cough

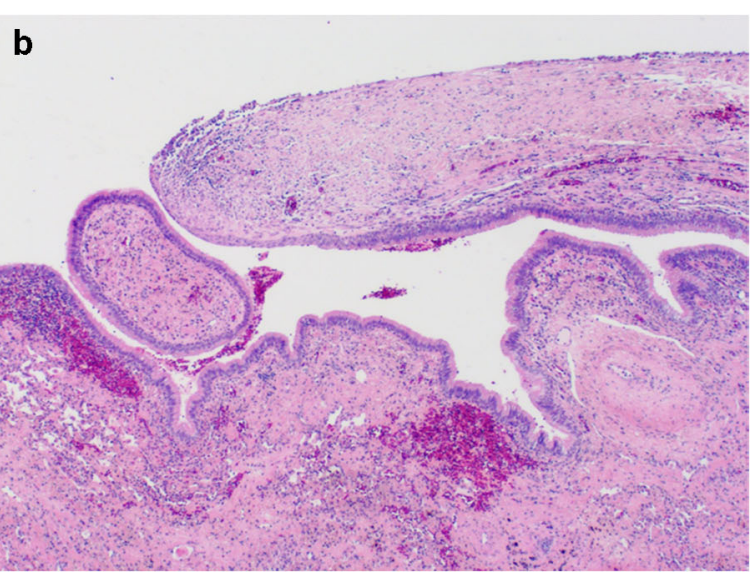

transitioning with ciliated respiratory epithelium (left) (reprinted with permission from the European Respiratory Journal)

may constitute a contributory factor in the development of pneumothorax in these patients [4].

The radiological features of COVID-19 infection have been described since early in the pandemic [7, 15], and several case reports and series describe the imaging features seen in the setting of pneumothorax and pneumomediastinum. Several cases of pneumothorax associated with cystic pulmonary lesions on chest $\mathrm{CT}$ in patients with COVID-19 have also been reported $[3,14]$; these cystic changes may result from resorption of consolidation [7], which may also result in pneumatocoele formation, as was found in our case. Pneumatocoele formation was reported in a recent case series by Mallick et al.- in two out of three patients who presented with pneumothoraces and were found to be SARS-CoV-2positive [1]. They postulate that prolonged inflammation may lead to the development of pneumatocoeles due to alveolar wall destruction of adjacent airspaces. Pneumatocoeles, which are defined as thin-walled, air-filled intraparenchymal cysts that develop secondary to localized bronchiolar and alveolar

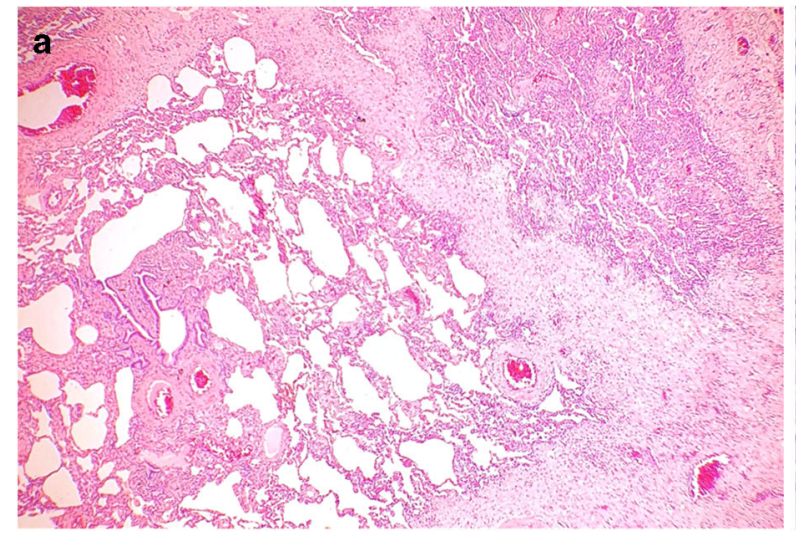

Fig. 3 a Background lung showing septal fibrosis with oedema and vascular congestion with alternating areas of aerated and collapsed lung (reprinted with permission from the European Resipratory

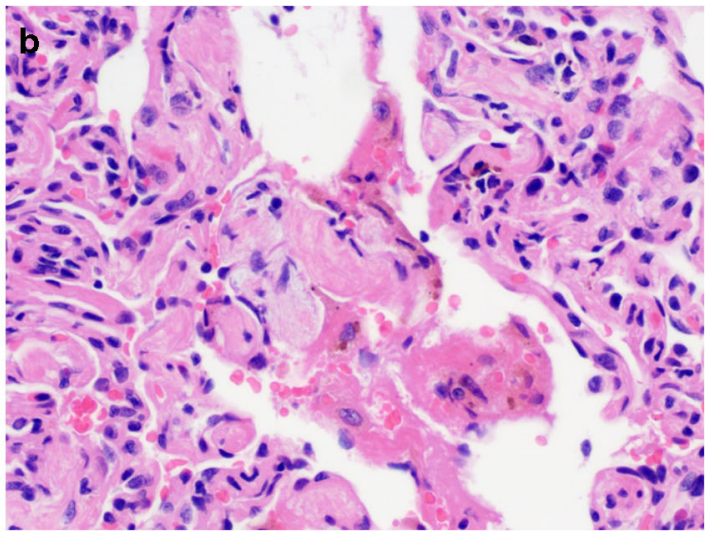

Journal). b High power view showing intra-alveolar fibrin and a fibromyxoid plug with focal haemosiderin deposition 
necrosis, are more commonly associated with bacterial pneumonia—notably S. aureus and S. pneumoniae [16]. We note a recently described case of co-infection of COVID-19 and tuberculosis in the absence of a history of immunodeficiency [17]. In our case, it is unclear whether the pneumatocoele that developed was a result of COVID-19 or the patient's prior bacterial pneumonia - or indeed a combination of the two.

In summary, we describe the case of a patient with COVID-19 infection complicated by pneumothorax (for which there is growing evidence of an association with COVID-19) and describe the histological features therein, namely, patchy parenchymal collapse and congestion, septal fibrosis and oedema, intra-alveolar fibromyxoid and fibrinous material, and features of pneumatocoele formation. Furthermore, this case — among others [18]—demonstrates that negative nasopharyngeal swabs prior to thoracic surgery do not definitively exclude COVID-19 infection and, as such, these surgical specimens should always be treated as potentially infectious with appropriate precautions taken at the cutup bench.

\section{Author Contributions}

- Draft of the manuscript: Pascale Capleton, Nairi Tchrakian

- Contribution and interpretation of histopathological data: Nairi

Tchrakian, Michael Sheaff, Konstantinos Giaslakiotis

- Contribution and interpretation of clinical data: William Ricketts,

Kelvin Lau, Stephanie Uys

- Contribution and interpretation of radiological data: Stephen Ellis

Data Availability Not applicable.

\section{Compliance with Ethical Standards}

Conflicts of Interest The authors declare that they have no conflicts of interests.

Ethics Approval Not applicable.

Consent to Participate Not applicable.

Consent for Publication This case - including Figs. 1b, $2 \mathrm{~b}$ and $3 \mathrm{a}$ - has been included in a case series which has recently been accepted for publication [13]. Permission was sought — and granted — from the European Respiratory Journal to include the images in question in this case report.

Code Availability Not applicable.

\section{References}

1. Mallick T, Dinesh A, Engdahl R, Sabado M. COVID-19 complicated by spontaneous pneumothorax. Cureus. 2020;12(7):e9104.

2. Aiolfi A, Biraghi T, Montisci A, Bonitta G, Micheletto G, Donatelli $\mathrm{F}$, et al. Management of persistent pneumothorax with thoracoscopy and blebs resection in Covid-19 patients. Ann Thorac Surg. 2020;110:e413-5.

3. Sun R, Liu H, Wang X. Mediastinal emphysema, giant bulla, and pneumothorax developed during the course of COVID-19 pneumonia. Korean J Radiol. 2020;21(5):541-4.

4. Ucpinar BA, Sahin C, Yanc U. Spontaneous pneumothorax and subcutaneous emphysema in COVID-19 patient: case report. J Infect Public Health. 2020;13(6):887-9.

5. Xiang C, Wu G. SARS-CoV-2 pneumonia with subcutaneous emphysema, mediastinal emphysema, and pneumothorax: a case report. Medicine (Baltimore). 2020;99(20):e20208.

6. Salehi S, Abedi A, Balakrishnan S, Gholamrezanezhad A. Coronavirus disease 2019 (COVID-19): a systematic review of imaging findings in 919 patients. AJR Am J Roentgenol. 2020;215(1):87-93.

7. Shi H, Han X, Jiang N, Cao Y, Alwalid O, Gu J, et al. Radiological findings from 81 patients with COVID-19 pneumonia in Wuhan, China: a descriptive study. Lancet Infect Dis. 2020;20(4):425-34.

8. von der Thusen J, van der Eerden M. Histopathology and genetic susceptibility in COVID-19 pneumonia. Eur J Clin Investig. 2020;50(7):e13259. https://doi.org/10.1111/eci.13259.

9. Zhang H, Zhou P, Wei Y, Yue H, Wang Y, Hu M, et al. Histopathologic changes and SARS-CoV-2 immunostaining in the lung of a patient with COVID-19. Ann Intern Med. 2020;172(9):629-32.

10. Xu Z, Shi L, Wang Y, Zhang J, Huang L, Zhang C, et al. Pathological findings of COVID-19 associated with acute respiratory distress syndrome. Lancet Respir Med. 2020;8(4):420-2.

11. Heinrich F, Sperhake JP, Heinemann A, Mushumba H, Lennartz M, Nörz D, et al. Germany's first COVID-19 deceased: a 59-yearold man presenting with diffuse alveolar damage due to SARSCoV-2 infection. Virchows Arch. 2020;477(3):335-9.

12. Tian S, Hu W, Niu L, Liu H, Xu H, Xiao SY. Pulmonary pathology of early-phase 2019 novel coronavirus (COVID-19) pneumonia in two patients with lung cancer. J Thorac Oncol. 2020;15(5):700-4.

13. Martinelli AW, Ingle T, Newman J, Nadeem I, Jackson K, Lane ND, et al. COVID-19 and pneumothorax: a multicentre retrospective case series. Eur Respir J. 2020;56:2002697.

14. Liu K, Zeng Y, Xie P, Ye X, Xu G, Liu J, et al. COVID-19 with cystic features on computed tomography: a case report. Medicine (Baltimore). 2020;99(18):e20175.

15. Bentivegna E, et al. Extremely severe case of COVID-19 pneumonia recovered despite bad prognostic indicators: a didactic report. SN Compr Clin Med. 2020;1-4. https://doi.org/10.1007/s42399020-00383-0.

16. Al-Saleh S, Grasemann H, Cox P. Necrotizing pneumonia complicated by early and late pneumatoceles. Can Respir J. 2008;15(3): 129-32.

17. Luciani M, et al. Coinfection of tuberculosis pneumonia and COVID-19 in a patient vaccinated with Bacille Calmette-Guerin (BCG): case report. SN Compr Clin Med. 2020;1-4. https://doi. org/10.1007/s42399-020-00601-9.

18. Bentivegna E, Sentimentale A, Luciani M, Speranza ML, Guerritore L, Martelletti P. New IgM seroconversion and positive RT-PCR test after exposure to the virus in recovered COVID-19 patient. J Med Virol. 2020. https://doi.org/10.1002/jmv.26160.

Publisher's Note Springer Nature remains neutral with regard to jurisdictional claims in published maps and institutional affiliations. 